\title{
Comercio informal, características e incidencia en la externalización de costos en la ciudad de Huaraz
}

\author{
Informal trading, characteristics and impact on externalization of cost in \\ Huaraz City \\ JoHn TARAZONA JiMÉNEZ ${ }^{1}$
}

\section{RESUMEN}

El trabajo de investigación analiza el comercio informal ambulatorio, sus características e incidencia en la externalización de costos en la ciudad de Huaraz, departamento de Áncash, Perú. Para el desarrollo de la investigación se aplicaron cuestionarios estructurados a muestras aleatorias, en tres zonas donde se concentra el comercio informal dentro de la ciudad de Huaraz. De los resultados se infiere la existencia de relación entre una condición de alta externalización de costos y el poco interés por la formalización; de manera inversa, en aquellos comerciantes informales cuya condición de externalización es baja, tienen mayor interés en formalizarse. Además se ha determinado los factores que contribuyen a una mayor externalización de costos en los comerciantes informales de la ciudad de Huaraz y, por tanto, poco interés hacia la formalización. Estos factores se han identificado como la edad del comerciante informal, el número de días que realiza la actividad de comercio informal, lugar donde adquiere la mercadería.

Palabras clave: externalización de costos; comercio informal; costos; ambulantes.

\section{ABSTRACT}

The research paper analyzes the ambulatory informal trading and its characteristics and impact on the externalization of cost in the city of Huaraz, Ancash department, Peru. For the development of the research, structured questionnaires have been tested to aleatory in three areas where informal trading is being set inside the city of Huaraz, from the results, it has been deduced that the relation existence between a condition

1 Universidad Nacional «Santiago Antúnez de Mayolo». Huaraz - Perú. 
of high externalization of cost and the low interest for the formalization; on the reverse order of those informal merchants whose externalization condition is low, these group are the ones that have a great interest for formalization, besides the contribution factors have been determined to a longer cost of externalization of informal merchants in the city of Huaraz and for the very low interest for formalization. The age of informal merchants, the number of days of informal trading activity and the place where the merchandise is received, have been identified on these factors.

Keywords: externalization of cost; informal trade; cost; street vendors.

\section{ICHIKLLACHAW}

Kay musyapakuyqa riqitsimantsik lluta rantikuq runakunapa kanqantam, imanaw Waras markachaw, Anqash suyuchaw chanintsaynin kanqantam. Kay musyapakuypaqqa tapukuykuna rurashqawanmi huk quchukashqa alyaturyakuna nishqan ayllukashqachaw, 03 sunachaw llapan lluta qatukuqkuna Waras markapa rurinchaw kayanqanchaw. Kaypiq nishwanmi tinkunmi atska waqta chanintsayninwan ichik alli rantikuy munayanqanwan; lluta qatukuqkunapaqqa ichikllam waqtachaw qatukuynin kan, manatsayqa alli qatukuymanmi tikrayta munayan. Kayqa Waraschaw waqtachaw qatukuqkunatam alliyaatsin, tsayraykurmi iñiyantsu alli qatukuyman. Kaychaw riqiqashqa lluta qatukuqpa watanta, llapan hunaq lluta qatukunqanta, maypiq llapan qatukuynin tarinqantam.

Pushaq shimikuna: waqtachaw chanintsaynin; lluta qatukuy; chaninkuna; lluta qatukuqkuna.

\section{INTRODUCCIÓN}

El presente trabajo de investigación busca contribuir al debate sobre el comercio informal urbano, incorporando una visión local del problema, donde se propone el análisis de los factores que influyen a que este problema se agudice, situación que viene sucediendo en los últimos años. El enfoque incorpora elementos del análisis institucionalista, que incluye el análisis de los actores dentro del mercado del comercio informal, la manera en que se relaciona bajo el enfoque de la racionalidad limitada de los agentes; esta actividad «forma parte del complejo entramado del mercado cuya racionalidad económica es la acumulación» (Magaña Hernández y Figueroa Díaz, 2013), por lo que los comerciantes son propensos a evitar pagos y/o no incorporar costos de producción en sus actividades, con el fin de apropiarse de esta parte de la renta «maximizando sus beneficios», hecho que en la práctica constituye la externalización de costos hacia el estado y la ciudadanía. Este sector tiene estímulos estructurales, pero también de carácter coyuntural, hecho que determina una compleja realidad. Como es sabido no se puede hablar de unidades económicas puramente formales y/o que no externalicen 
de alguna manera algunos costos; sin embargo, es importante evaluar aquellas unidades que externalizan mayores costos, como en el comercio informal. Recalcamos la importancia del fenómeno de la informalidad. "El sector subcapitalizado no tiene burocracia centralizada de registro y rastreo que ocupa el centro de la sociedad formal" (Arbaiza Fermini 2008) tema de interés para los que diseñan las políticas públicas. El trabajo ha empleado un modelo probabilístico para determinar aquellos factores que determinan la condición de alta externalización, y desinterés por formalizarse.

\section{MATERIALES Y MÉTODOS}

En cuanto al tipo de investigación, es aplicada y correlacional, de diseño trasversal, no experimental. La población de estudio se encuentra constituida por el número de comerciantes informales de la ciudad de Huaraz, 1911. La muestra empleada para el estudio está constituida por 184 comerciantes (véase la fórmula aplicada).

$n=\frac{z^{2} * p * q * N}{E^{2} *(N-1)+Z^{2} * P * Q}$

Con la información recogida en la encuesta estructurada, a una muestra representativa se ha aplicado el tratamiento econométrico de pronóstico probabilístico, según el cual el modelo de regresión logística binaria múltiple aplicado es:

$p=\frac{1}{1+e^{-z}}$

Dónde:

$\mathrm{Z}=\mathrm{B} 1 \mathrm{X}_{1}+\mathrm{B} 2 \mathrm{X}_{2}+\mathrm{B} 3 \mathrm{X}_{3}+\mathrm{B} 4 \mathrm{X}_{4}+\mathrm{B} 5 \mathrm{X}_{5}+\ldots$

\section{RESULTADOS}

A continuación se muestra el proceso de estimación del modelo de probabilidad para los comerciantes informales que externalizan costos, además se ha realizado una comparación de la probabilidad de externalizar altos costos con respecto a los grupos que no tienen interés por formalizarse y obtienen bajos ingresos.

Tabla 1. Externalización de costos

\begin{tabular}{lrr}
\hline Externalización de costos & Frecuencia & \multicolumn{2}{c}{ Porcentaje } \\
\hline Baja & 72 & 39,1 \\
Alta & 112 & 60,9 \\
Total & 184 & 100,0 \\
\hline
\end{tabular}


El análisis de acuerdo a los ítems de «pagos» a ciertos servicios básicos, como a seguridad y limpieza pública, nos han permitido generar la tabla presentada anteriormente, en donde podemos apreciar que el 39,1\% de los comerciantes ambulantes genera una baja externalización de costos; quiere decir que realiza algunos pagos o tasas; sin embargo, más del 60\% de los comerciantes ambulantes generan una alta externalización de los costos, es decir, evitan el pago de algunos conceptos vinculados al costo del comercio y esto genera una externalización de costos hacia el estado y finalmente a la población.

Tabla 2. Ingreso promedio mensual por comercio ambulatorio en grupos que externalizan costos

\begin{tabular}{lrrrr}
\hline $\begin{array}{l}\text { Externalización } \\
\text { de costos }\end{array}$ & Media & N & Desv. típ. & Mediana \\
\hline Baja & 707,9 & 72 & 283,7 & 678 \\
Alta & 658,4 & 112 & 328,3 & 600 \\
Total & 677,8 & 184 & 311,8 & 678 \\
\hline
\end{tabular}

Para hacer la comparación de grupos de personas que tienen mayores ingresos y que externalizan altos costos, lo primero que debemos conocer es que si existe diferencia entre ambos grupos con respecto a los ingresos, como podemos apreciar en la tabla anterior, la media de los ingresos de grupos que externalizan altos costos es de 658 nuevos soles, mientras que los que externalizan menos es de 707 nuevos soles aproximadamente, es decir, relativamente los que externalizan bajos costos tienen mayores ingresos; sin embargo esta diferencia no es muy significativa como podemos observar en el cuadro de diferencia de medias, donde la prueba de significancia es mayor que 0,005; es decir, el análisis T de diferencia de medias nos indica que no existe diferencia significativa entre ambas medias.

Tabla 3. Prueba t para diferencia de medias

\begin{tabular}{|c|c|c|c|c|c|}
\hline & $\begin{array}{c}\text { Suma de } \\
\text { cuadrados }\end{array}$ & gl & $\begin{array}{c}\text { Media } \\
\text { cuadrática }\end{array}$ & F & Sig. \\
\hline Inter-grupos & 107255,8 & 1 & 107255,8 & 1,10 & 0,29 \\
\hline Intra-grupos & 17680145,2 & 182 & 97143,7 & & \\
\hline Total & 17787401,0 & 183 & & & \\
\hline
\end{tabular}




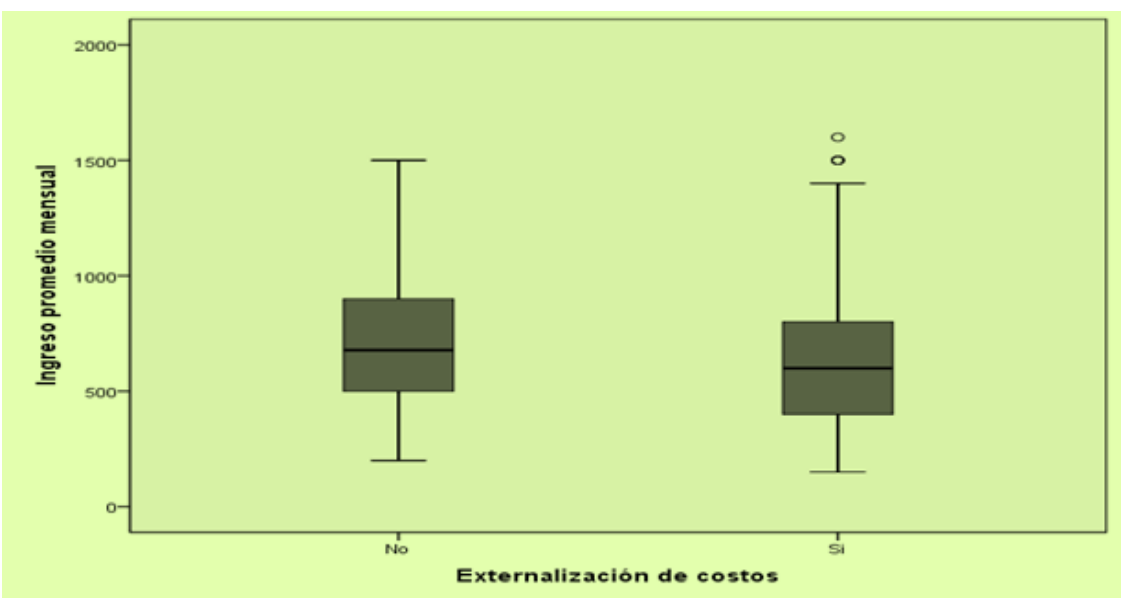

Figura 1. Ingresos y externalización de costos

La figura 1, nos muestra la distribución de los ingresos en ambos grupos, donde podemos apreciar que son iguales relativamente, con una media menor en el grupo que sí externaliza costos.

Tabla 4. Prueba de normalidad del ingreso promedio mensual

\begin{tabular}{ccccccc}
\hline \multirow{2}{*}{$\begin{array}{c}\text { Pruebas de normalidad } \\
\end{array}$} & \multicolumn{2}{c}{ Kolmogorov-Smirnova } & \multicolumn{3}{c}{ Shapiro-Wilk } \\
& Estadístico & gl & Sig. & Estadístico & gl & Sig. \\
\hline $\begin{array}{c}\text { Ingreso promedio } \\
\text { mensual }\end{array}$ & 0,146 & 184 & 0,000 & 0,939 & 184 & 0,000 \\
\hline
\end{tabular}

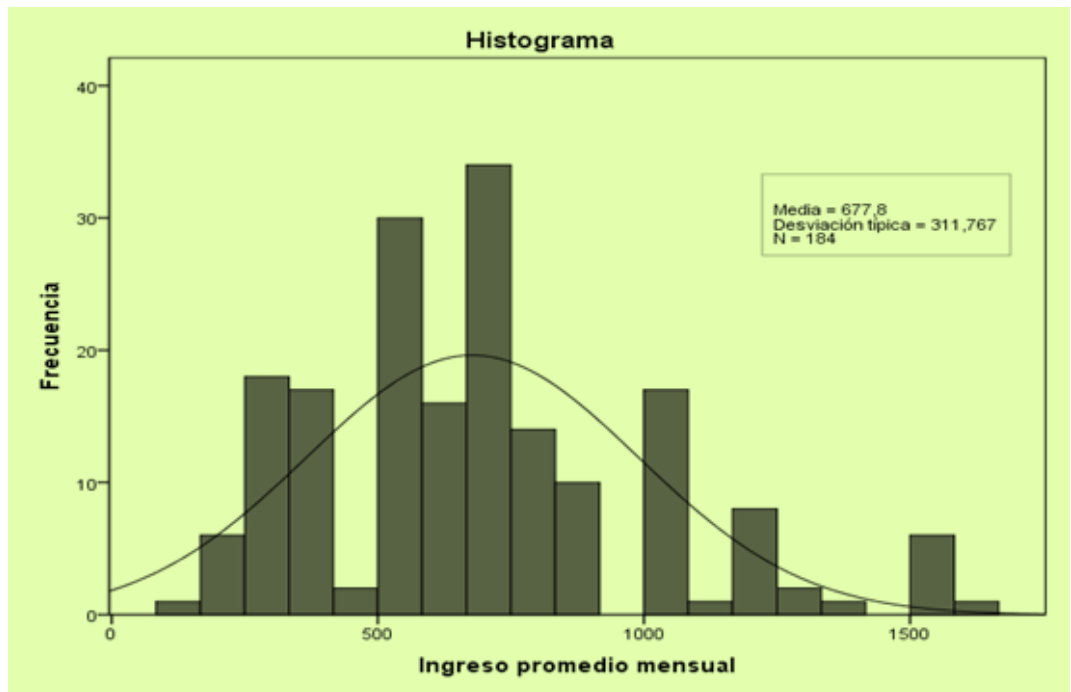

Figura 2. Barras con curva de la normal 
La prueba y el gráfico nos muestran la normalidad de nuestros datos con respecto al ingreso promedio mensual que tienen los comerciantes ambulantes. En este caso, el valor de significancia $\mathrm{p}<0.005$, por tal motivo podemos afirmar que los datos del ingreso promedio mensual se distribuyen de manera normal; esto contribuirá al análisis para poder generar un mejor pronóstico de los factores que inciden en la alta externalización de costos.

Tabla 5. Interés por formalizarse en grupos que externalizan costos

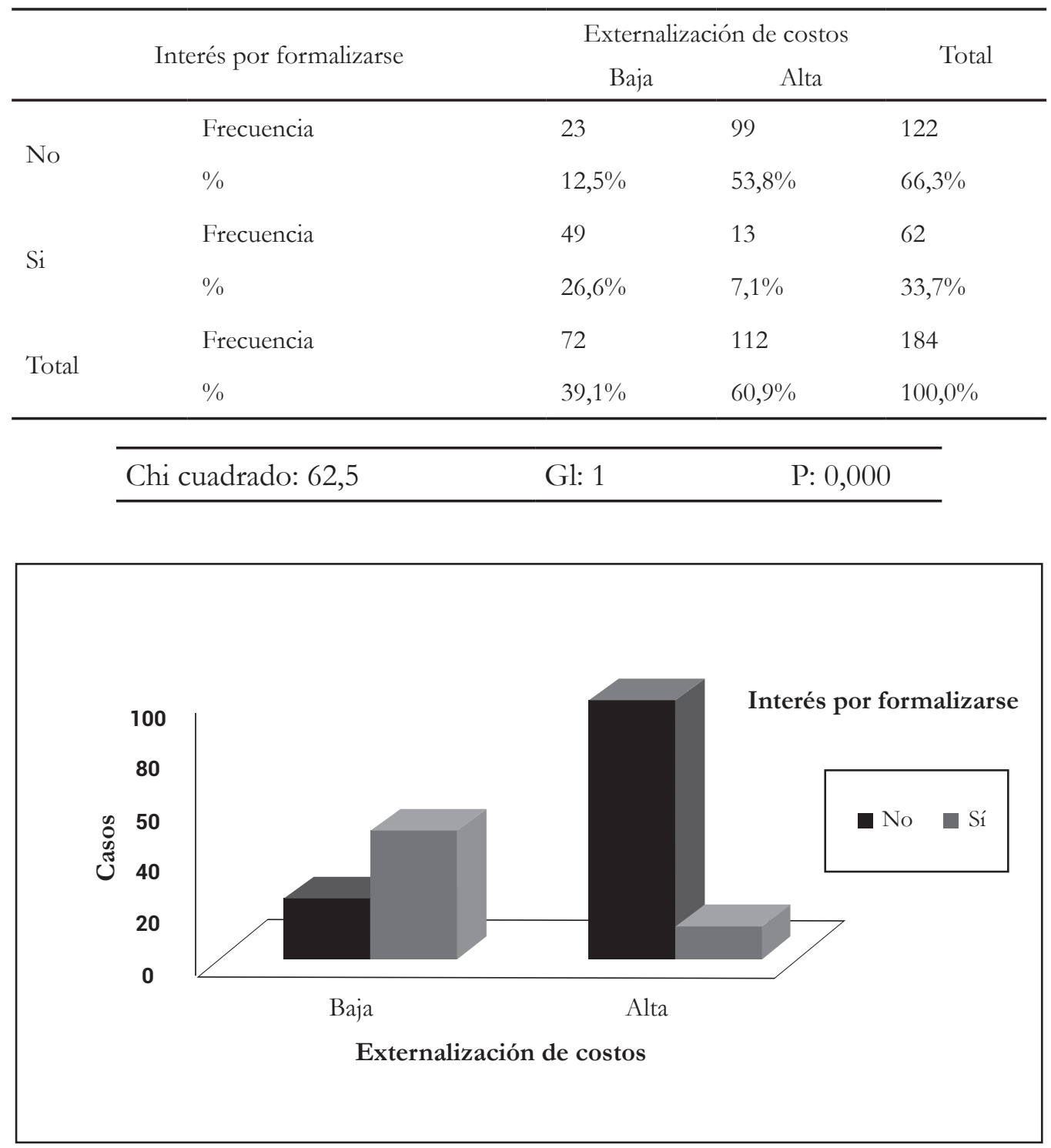

Figura 3. Externalización de costos vs Interés de formalizarse 
Podemos observar en el gráfico, la justificación de la prueba estadística Chi cuadrado, donde la interpretación nos indica que existe una relación positiva entre la externalización de costos y el interés por formalizarse. Es así que en grupos que tienen baja externalización de costos tienen mayor interés por formalizarse mientras que el grupo que tiene alta externalización de costos tiene un bajo interés por formalizarse o tiene poco interés.

El modelo propuesto se presenta en la tabla $\mathrm{N}^{\circ}$ 6. Asimismo, se ha generado un modelo biprobabilístico, es decir, se ha hecho una comparación entre los factores que inciden en la externalización de generar altos costos con respecto al hecho de no generar buenos ingresos, así como la falta de interés por formalizarse.

Tabla 6. Modelo de probabilidad de generar alta externalización de costos

\begin{tabular}{|c|c|c|c|c|c|c|c|c|c|c|}
\hline \multirow{3}{*}{ Factores } & \multirow{2}{*}{\multicolumn{2}{|c|}{$\begin{array}{l}\text { Probabilidad de gene- } \\
\text { rar alta externalización } \\
\text { MODELO } 1\end{array}$}} & \multicolumn{8}{|c|}{$\begin{array}{l}\text { Probabilidad de generar alta externalización } \\
\text { MODELO BI PROBABILISTICO }\end{array}$} \\
\hline & & & \multicolumn{4}{|c|}{ Ingresos altos Ingresos bajos } & \multicolumn{2}{|c|}{$\begin{array}{l}\text { Intención de } \\
\text { formalizar }\end{array}$} & \multicolumn{2}{|c|}{$\begin{array}{l}\text { Intención de } \\
\text { no formalizar }\end{array}$} \\
\hline & B & Sig. & B & Sig. & B & Sig. & B & Sig. & B & Sig. \\
\hline $\begin{array}{l}\text { Edad mayor o igual } \\
\text { a } 44 \text { años }\end{array}$ & $-0,803$ & 0,031 & & & $-1,385$ & 0,046 & & & $-1,347$ & 0,026 \\
\hline Casado & 0,998 & 0,017 & & & & & & & & \\
\hline $\begin{array}{l}\text { Días de trabajo } \\
\text { menores a } 5\end{array}$ & 0,994 & 0,016 & & & 2,373 & 0,004 & & & & \\
\hline $\begin{array}{l}\text { El comercio es un } \\
\text { negocio atractivo }\end{array}$ & 1,475 & 0,001 & & & & & & & & \\
\hline $\begin{array}{l}\text { Interés por forma- } \\
\text { lizarse }\end{array}$ & $-2,804$ & 0,000 & $-2,079$ & 0,000 & $-3,894$ & 0,000 & & & & \\
\hline $\begin{array}{l}\text { Adquiere la merca- } \\
\text { dería directamente } \\
\text { de los agricultores }\end{array}$ & $-1,152$ & 0,007 & & & $-2,677$ & 0,001 & & & $-1,472$ & 0,016 \\
\hline $\begin{array}{l}\text { Es comerciante por- } \\
\text { que no consiguió } \\
\text { otro empleo }\end{array}$ & & & 1,684 & 0,001 & & & & & & \\
\hline $\begin{array}{l}\text { Es comerciante } \\
\text { porque se genera } \\
\text { dinero en forma } \\
\text { diaria }\end{array}$ & & & & & 2,619 & 0,000 & & & 3,366 & 0,000 \\
\hline $\begin{array}{l}\text { Disposición a cam- } \\
\text { biar de actividad }\end{array}$ & & & & & $-2,026$ & 0,016 & & & & \\
\hline $\begin{array}{l}\text { Es comerciante por } \\
\text { la flexibilidad de los } \\
\text { horarios }\end{array}$ & & & & & & & $-1,727$ & 0,000 & & \\
\hline $\begin{array}{l}\text { Prueba de Hosmer } \\
\text { y Lemeshow }\end{array}$ & 0,075 & & 0,1 & & 0,1 & & 0,0 & 00 & 0,8 & 91 \\
\hline $\begin{array}{l}\text { Probabilidad de } \\
\text { pronóstico }\end{array}$ & 85,3 & & 79 & & 90 & & 80 &, 6 & 88 &, 5 \\
\hline
\end{tabular}


El Modelo 1 representa los resultados obtenidos en la aplicación del modelo logístico, que no es más que la probabilidad de generar alta externalización de costos dados los indicadores planteados en nuestro estudio, determinando que los factores que contribuyen a generar una probabilidad positiva para la alta externalización de los costos es tener una edad no mayor a los 44 años, ser casado, trabajar menos días de la semana. Quienes consideran que es un negocio atractivo el comercio ambulatorio, no tienen interés por formalizarse, y no adquieren la mercadería de manera directa de los productores sino de mayoristas locales.

El modelo biprobabilístico, es un modelo condicionado a ciertos factores. En el caso de nuestro estudio se ha comparado con dos factores, uno de ellos es generar ingresos altos o bajos, mientras que otro es la intención de formalizar o no formalizar.

\section{DISCUSIÓN}

Los resultados obtenidos en el análisis nos muestran que existen diferentes niveles de externalización de costos. De acuerdo al marco teórico externalizan costos aquellas unidades económicas cuyos costos de producción no son abonados en su totalidad por estas unidades, y con frecuencia en las economías informales, y específicamente el comercio informal que utiliza áreas públicas para su localización no incorporan un conjunto de costos, emplean infraestructura construida a costa de la colectividad (Wallerstein, 2003), que si son incorporados por las unidades económicas formalizadas, se ven afectados por esta externalización de costos (Rocha, Sánchez y García, 2016). Para el análisis, reconocemos en función del argumento teórico que todas la unidades de comercio informal externalizan costos; sin embargo no con la misma dimensión. Existen algunos comerciantes informales que externalizan en mayor o menor proporción, trasmitiendo estos costos al estado y a la sociedad, también en diferentes niveles, para identificar estos niveles de externalización en el estudio se ha tomado un conjunto de costos asociados a la actividad económica de manera que se observa que el $60.9 \%$ de los comerciantes informales estudiados tiene una alta externalización de costos, pues casi no cubren ningún costo asociado a la actividad, mientras que solo el 39.1\% de los comerciantes se puede considerar que tiene una baja externalización de costos.

En el segundo análisis se incorporan dos factores importantes para la discusión y búsqueda de soluciones al problema del comercio informal, la relación probabilística entre la alta externalización de costos con los niveles de ingresos y el interés por la formalización.

Los comerciantes ambulantes que tienen menos de 44 años, trabajan menos de cinco días a la semana, no tienen interés por formalizarse, no adquieren la mercadería directamente de los productores sino de los mayoristas locales, y además opinan que el 
comercio ambulatorio es una actividad que genera dinero en forma diaria e inmediata, y no están dispuestos a cambiar de actividad son las características de los comerciantes ambulantes que generan bajos ingresos y además generarían un nivel alto de externalización de costos.

\section{CONCLUSIÓN}

El comercio informal en la ciudad de Huaraz se caracteriza por altos niveles de externalización de costos, debido que la mayoría de comerciantes evita pagar algunos costos asociados a estas actividades económicas. Este hecho respondería por un lado a su carácter de subsistencia, pero también a conductas vinculadas a la apropiación de recursos para rentabilizar estas externalidades (Rossi, 2008).

En cuanto al proceso de formalización se ha observado el poco interés por llegar formalizarse $(75 \%)$ teniendo como característica principal la intención de evitar el pago de algunos costos relacionados a la formalización de la actividad.

El estudio identifica un conjunto de factores que influyen en la probabilidad de una alta externalización de costos vinculada con los bajos niveles de ingresos y el poco interés de formalizarse, de manera que estarían explicados en orden de importancia por los siguientes: que los productos del campo se adquieran a intermediarios, y no de manera directa influye para que exista una alta externalización; el hecho de que el comerciante valore la generación diaria de ingresos estimula una mayor externalización de costos, además de la edad del comerciante informal, si es más joven tiende a una mayor externalización de costos.

\section{AGRADECIMIENTOS}

A la Universidad Nacional «Santiago Antúnez de Mayolo», por el financiamiento recibido para la realización de la presente investigación.

\section{REFERENCIAS BIBLIOGRÁFICAS}

Arbaiza, Lydia. 2008. Economía informaly capital humano en el Perú. Lima: Ediciones ESAN.

Magaña, D. M. \& Figueroa, L. (2013). « Reflexiones en torno al fenómeno del mercado informal y la libertad de comercio ». Alegatos - Revista Jurídica de La Universidad Autónoma Metropolitana, 515-538.

Rocha , R.; Sánchez, F. \& García, L. (2016). Ventas callejeras y espacio público: efectos sobre el comercio de Bogotá. Desarrollo y Sociedad, 245-268. 
| John Tarazona

Rossi, F. J. (2008). Desarrollo de la economía informal. Análisis Económico, 23(52), 133 158.

Wallerstein, I. (2003). La decadencia del poder estadounidense. México: Editores Independientes.

Fecha de recepción: 10 de febrero 2016

Fecha de aceptación: 31 de mayo 2016

\section{Correspondencia}

John Joseph Tarazona Jiménez

johntarazona@gmail.com 